\title{
REMINISCENCE: A MOBILE INTELLIGENT System to Assist Dementia Patients
}

\author{
Joan Wang ${ }^{1}$, Shiyu Feng ${ }^{2}$, Amanda Zhu ${ }^{3}$, Alina Yue ${ }^{4}$, Fangyan Zhang \\ Yu Sun ${ }^{6}$ \\ ${ }^{1}$ Aliso Niguel High School, Aliso Viejo, CA 92656 \\ ${ }^{2}$ Arnold O. Beckman High School, Irvine, CA, 92602 \\ ${ }^{3}$ Portola High School, Irvine, CA, 92618 \\ ${ }^{4}$ Crean Lutheran High School, Irvine, CA, 92620 \\ ${ }^{5}$ ASML, San Jose, CA, 95131 \\ ${ }^{6}$ California State Polytechnic University, Pomona, CA, 91768
}

\begin{abstract}
Since people pay more attention to health issues, how to effectively diagnose potential diseases becomes gradually important in that most of us are not physical experts. This paper proposes a single collective app that offers solutions to both potential and diagnosed patients based on machine learning. Users can use the app to evaluate their status by uploading their documents. It is helpful to tell users the potential issues concerning health in advance.
\end{abstract}

\section{KEYWORDS}

Machine Learning, disease diagnose, dementia prevention

\section{INTRODUCTION}

Dementia is any decline in recognition that is significant enough to interfere with independent, daily functioning. Dementia is best characterized as a syndrome rather than as one particular disease [1]. Thus, doctors identify neurodegenerative diseases and conditions that cause dementia-like symptoms as dementia. Neurodegenerative diseases occur when neurons lose function over time and ultimately lead to cell death [2]. Since cells in the central and peripheral nervous systems mostly cannot multiply or be replaced, the damaged cells remain impaired until its death. Therefore, neurodegenerative diseases are progressive and incurable.

While the number of people diagnosed with dementia has increased in recent years, treatments and medications for the symptoms have advanced. The main goal of professional treatments is to serve as a buffer to improve dementia symptoms. An accurate diagnosis and medications can boost levels of chemical messengers in the nervous system to help with areas like memory and judgement. Although medical advancements certainly benefit patients, a better solution to the escalating number of people showing dementia symptoms is to reduce the risk of developing dementia when getting older.

A majority of middle-aged people or elderly without dementia are made aware of the statistics and the situation. It is common knowledge among families that a healthier lifestyle can lessen the possibility of developing dementia. Websites such as the Alzheimer's Society provides complete guide to what people should or should not do for a stronger mind and body. Seniors are 
encouraged by their family members or caretakers to socialize more and to actively train themselves through simple exercises. Activities mentioned above are valid and are used to achieve the same objective. In addition, for those who are already experiencing dementia symptoms, technological methods such as home monitoring devices and reminder messages aid the caretakers when they are not around. In gratitude to specialized equipment, caretakers could rest assured most of the time. However, there is not a single collective app that offers solution to both potential and diagnosed patients.

\section{Challenges}

\subsection{Challenge 1: Inconsistent Activity on the Application}

The first of many challenges to address are the inconsistency of the user utilizing the game section of the application. In an ideal situation, the user is expected to play the games an equal number of times every single day to ascertain the accuracy of the statistic. However, such a model cannot be achieved in the real-life situation. Here are some of the variables to consider: the place at which the user live, how much the user uses the phone, and if the user remember to open the app. if elders live in an area with plenty of seniors like them who are mostly retired and have abundant free time, then there is no need to worry about their daily socializing events. However, unless they reside designated seniors' areas or with their family, seniors have lesser chance to meet other people. Typically, among average American adults, more socialization means less phone screen time; it could be assumed the same for seniors. Each user is a different case therefore each user spends an unpredictable amount of time of his or her phone and the app.

\subsection{Challenge 2: User Progress}

Medically, one's brain cognitive function cannot be measured with certainty. The normally accepted standard is to identify dementia by stages. For users without dementia, there is no scale for them to see their progress with a perceptible system. Without a workable system, neither the users nor their caretakers can identify problems early on. Although dementia can be identified through certain physical symptoms such as mood swings and reduced concentration, it be prove difficult if family members do not live close to users.

\subsection{Challenge 3: Family Participation}

Family participation is important whether the senior has or does not show dementia symptoms. It counts as a form of encouragement for elders to keep living a healthier lifestyle and exercising with regularity. Families and caretakers usually are aware of several potential risks the patients might face while living away from them: inability to contact and getting lost. There is also this worry that people with dementia will forget to use the application and results in wasting all previous efforts.

\section{Solution}

The best solution is a simple app that offers all of the components that can be useful to deterring the growth of dementia. We will use data generated graph to accurately display progress and offer information that will improve the overall health of seniors (Figure 1). 


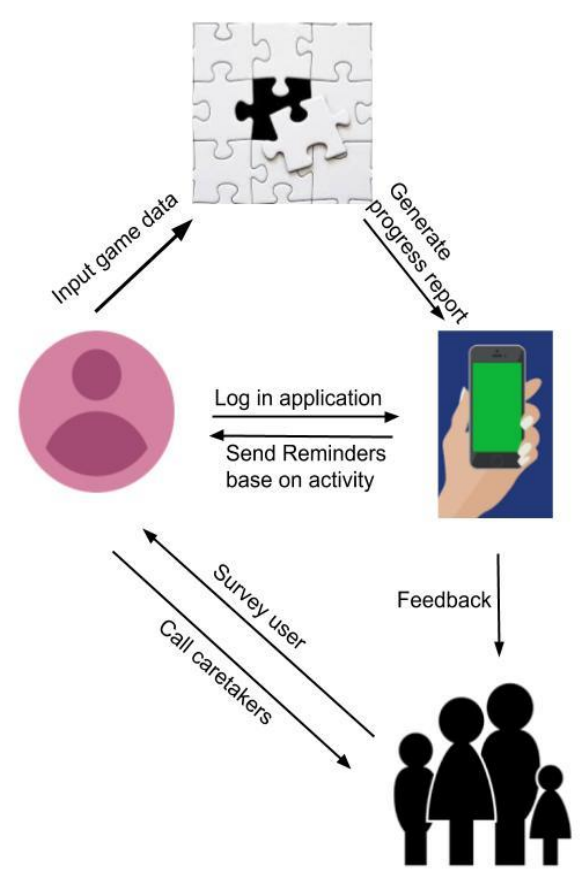

Figure 1: Overview of the Application

\subsection{Automatic Daily Sign in When User Meet Certain Requirements, Notify Caretakers}

Research suggests that spaced practices are more efficient that amassing a large amount of attention in one time period[3] Thus, it is of high significance that seniors In order for seniors who do not have dementia to come back daily and utilize the functions on the app, there needs to be incentives that could provide benefits to them. The mobile application could set up a daily sign in system at which the system automatically registers the user's daily activity on the application. Because each individual has its own schedule, we could assume that the amount of phone usage could also be different. According to each individual's average cell phone usage time, the application can generate an estimated amount of time that each individual should spend in the application (i'm not sure how). Each completed day could equal to one token and the tokens can be the currency at which new games or recipes can be "unlocked". Since this is targeted to seniors who do not yet show dementia symptoms, we can assure that they will not forget to come back if there are incentives present.

\subsection{Utilize Users' Statistics to Clearly Demonstrate the Progress}

Another problem discussed was the fact that there is no way that progress can be shown. However, by using the games data in the mobile application, the system can generate a line graph that demonstrates the average progress that each user displayed. We could predetermine the point systems in games and the mobile application calculates the final results that will be shown on the graph. We could also predetermine the ranges that identify with each stage of dementia. This could serve as a precaution for those who do not have dementia and also to notify caretakers if one's dementia stages have changed. 


\subsection{Family App That Connects the Caretaker with User}

Family involvement in dementia patients include various benefits, especially for long-term care patients, family involvement can reduce the risk of infection and hospitalization [4]. Despite the advantages of frequent visits and communal social engagements, there are always times where families and caretakers don't have the availability to visit. Due to this, one method to substitute for the lack of family members is through a complimentary mobile application designed for families and caretakers. For the security of dementia patients, it would be best if relatives are aware of his or her whereabouts. In case of emergencies and in cases where certain phone numbers are not pre-saved, the complementary application can aid with all the mentioned situations. Through real-time tracking, the complementary application is able to determine the location of users and to ensure their safety. The messaging system function in a unidirectional way; the family member or caretaker can send texts on the application to remind certain activities.

\section{EMPIRICAL RESUltS}

In this data, the computer randomly generated a list of points that would model the points earned in the game section of the application (Figure 2). The system than creates a scatter plot with a best fit line that most accurately represents the tendency of the user from daily activities on the application.

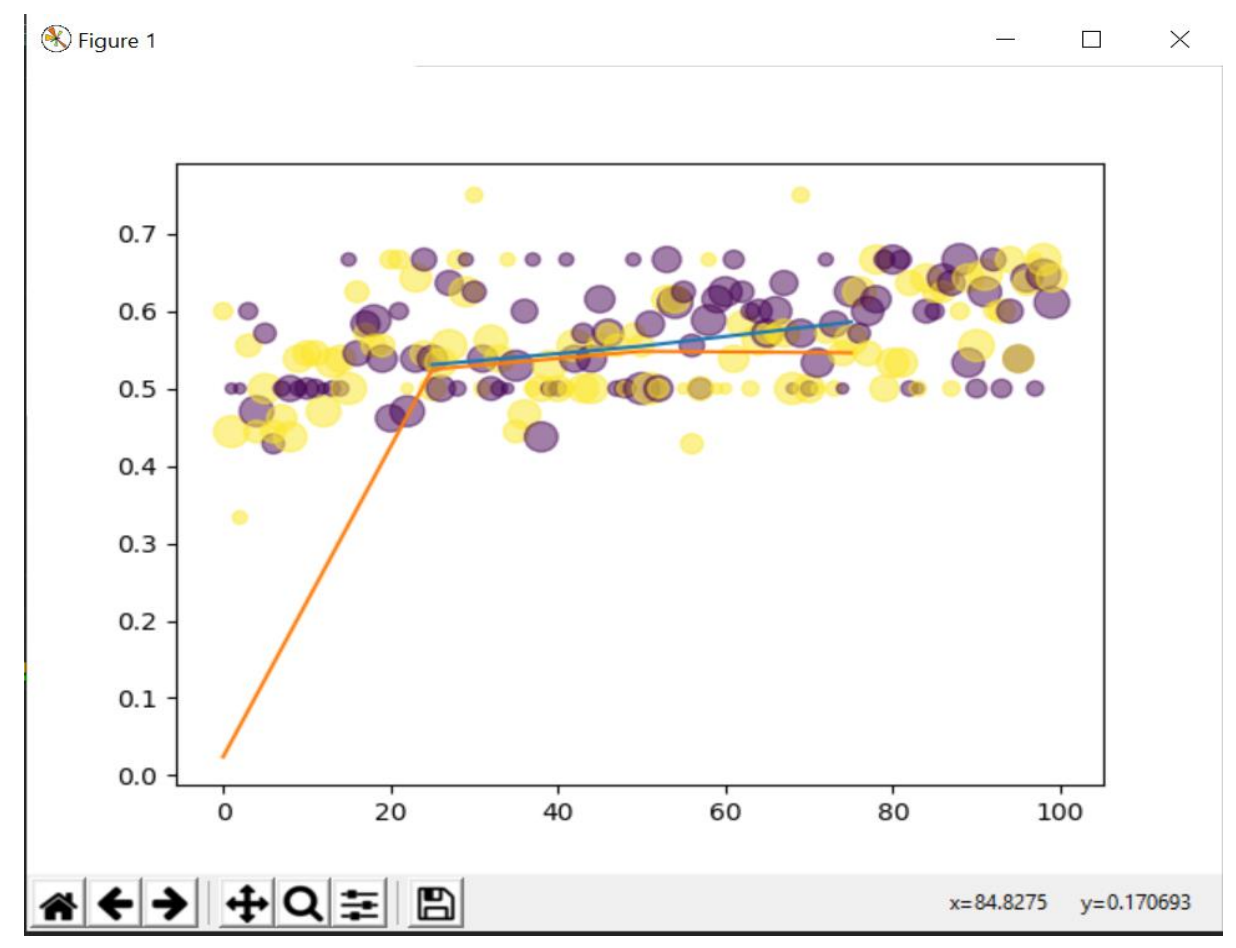

Figure 2: Scatter plot of empricial results

\section{RELATED WORK}

The world is garnering its attention on the gradual increase of dementia patients and the impact the condition has on families. Taking care of dementia patients of varying stages can take a huge amount of time and effort and it also stress financial burdens on the families. Therefore, prevention is by far the smartest to reduce such strain on all parties. There are papers that suggest 
prevention starting in childhood, starting from more childhood education, exercise, and retaining social engagement [5]. Since hypertension correlates with cognitive decline and dementia, treating hypertension in middle aged and older people can reduce the risk or delay the coming of dementia. Recently, antihypertensive treatments can be seen as a way toward dementia prevention. Results from therapeutic trials of two antihypertensive drugs suggest in the prevention of cognitive decline [6].

Other independent factors such as strokes and concussions also contribute to the acceleration of the development of dementia. Stratification by age could help to identify which intervention contributes most in which age-range, taking into account the age-dependent relationship for several vascular risk factors [7].

\section{CONClusions And Future Work}

In this paper, we aim to provide an automated and customizable development solution for the dementia prevention and deceleration community while supplying an application that keeps the health conditions of the users as its first priority. Due to the global population that has the same goal, mobile application that can assist with dementia symptoms could make a huge impact if presented correctly. We currently have the prototype for the application but needs further improvements on testing games efficiency and giving out information that require more professional guidance. As in our future work, we will expand it to include more machine generated data that can estimate daily time spent on application and programming more games that would work as incentives for users.

\section{REFERENCES}

[1] Gale, Seth A, Et Al. “Dementia.” The American Journal Of Medicine, Oct. 2018

[2] "Neurodegenerative Diseases." National Institute Of Environmental Health Sciences, U.S. Department Of Health And Human Services, Www.Niehs.Nih.Gov/Research/Supported/ Health/Neurodegenerative/Index.Cfm.

[3] Steward, Melissa M, Et Al. "Neural Regeneration." Current Topics In Microbiology And Immunology, 2013

[4] "Dementia - Neurologic Disorders." Merck Manuals Professional Edition, Merck Manuals, Www.Merckmanuals.Com/Professional/Neurologic-Disorders/Delirium-And-Dementia/Dementia.

[5] "Dementia." Mayo Clinic, 19 Apr. 2019, Www.Mayoclinic.Org/DiseasesConditions/Dementia/Diagnosis-Treatment/Drc-20352019.

[6] Maresova, Petra, Et Al. "Technological Solutions For Older People With Alzheimer's Disease: Review.” Current Alzheimer Research, Sept. 2018

[7] Kang, Sean. "Spaced Repetition Promotes Efficient And Effective Learning: Policy Implications For Instruction.” Policy Insights From The Behavioral And Brain Science, 2016

[8] Cohen, Lauren W, Et Al. "Dementia In Relation To Family Caregiver Involvement And Burden In Long-Term Care." Journal Of Applied Gerontology: The Official Journal Of The Southern Gerontological Society, Aug. 2014

[9] Cheng, Sheung-Tak. "Dementia Caregiver Burden: A Research Update And Critical Analysis." Current Psychiatry Reports, 10 Aug. 2017 
[10] Livingston, Sommerlad, Et Al. “Dementia Prevention, Intervention, And Care.” 20 July 2017.

[11] Hanon, Forette, "Prevention Of Dementia: Lessons From SYST-EUR And PROGRESS." Journal Of The Neurological Sciences, Elsevier, 29 Sept. 2004, Www.Sciencedirect.Com/Science/ Article/Abs/Pii/S0022510X04002977.

[12] Ramos-Cejudo, Jaime, Et Al. "Traumatic Brain Injury And Alzheimer's Disease: The Cerebrovascular Link.” Ebiomedicine, Elsevier, Feb. 2018.

[13] Richard, Andrieu, Et Al. "Methodological Challenges In Designing Dementia Prevention Trials The European Dementia Prevention Initiative (EDPI)." Journal Of The Neurological Sciences, Elsevier, 18 July 2012, 